\title{
Intelligent Collaborative Support System for AHP-Group Decision Making
}

\author{
Gang KOU ${ }^{1}$, Xiangrui CHAO ${ }^{2}$, Yi PENG ${ }^{2}$, Liang XU ${ }^{1}$, Yang CHEN ${ }^{1}$ \\ ${ }^{1}$ School of Business Administration, Southwestern University of Finance and Economics, \\ No.555, Liutai Ave, Wenjiang Zone, Chengdu, 611130, China \\ e-mail: kougang@swufe.edu.cn \\ ${ }^{2}$ School of Management and Economics, University of Electronic Science and Technology of China, \\ No.2006, Xiyuan Ave, West Hi-Tech Zone, Chengdu, 611731, China \\ Corresponding author: Yi Peng, e-mail: pengyi@uestc.edu.cn
}

\begin{abstract}
Analytic hierarchy process group decision making obtains the group's collective preference relationship based on the pair-wise comparative matrix of each decision maker by means of some aggregation rules, such as the aggregation of individual judgments and the aggregation of individual priorities. Then, consensus-reaching models are employed to help decision makers improve the consensus degree when the results are undesirable. A new preference aggregation method is proposed to integrate individual preference, which occurs when the group preference is nearest to all the individual priority vectors with weights by means of different optimization models. We further developed intelligent consensus-reaching model by adjusting the individual pair-wise comparison matrix in order to improve the acceptable individual priorities compared to the proposed group preference by the optimization models. The methods can directly improve a consensus-reaching degree without interactive preference modifications base on simple group preference aggregation method with row geometric mean. Some illustrative examples are examined to demonstrate the proposed models for application.
\end{abstract}

Keywords: T Group decision making; Row geometric mean; Consensus reaching; Optimization method.

\section{Introduction}

The analytic hierarchy process (AHP) was originally proposed by Saaty (1977, 1980, 1986) and is widely applied in decision making and management practice. The procedure obtains priorities based on multiple actors, scenarios, and criteria. The AHP is also frequently used in group decision making (GDM), which employs the AHP to answer group decision making questions due to its simplicity, power, and compatibility (Saaty 1989). For the AHP-GDM, each decision maker provides an individual pair-wise comparison matrix (PCM) in every local context of the hierarchy, and then several aggregation approaches are applied to obtain the group preference from the individual pairwise comparison matrices. The decisionmaking procedure in the AHP-GDM can be divided into 3 stages (Chiclana 2008, Dong et al. 2015): priority vector derivation, preference aggregation, and consensus reaching.

\section{Priority vector derivation}

The existing methods for the priority vector derivation from the PCM include the eigenvector method (EV) (Saaty, 1977), eighted least squares method (WLS)
(Chu, Kalaba, \& Spingarn, 1979), and logarithmic least squares method (LLS) (Crawford \& Williams, 1985), which obtain priority by optimization. The cosine maximization method (Kou \& Lin, 2014) derivates priority based on the maximum cosine similarity. Saaty (1980) proposed the consistency ratio (CR) for the consistency measurement. Crawford (1987) studied another consistency index using the geometric mean method. Peláez and Lamata (2003) described a consistency measurement method using the determinant of a PCM. Erguet al. (2011) and Kou et al. (2016) proposed an induced bias matrix to measure and improve the consistency of a PCM. Lin, Kou, and Ergu (2013b, 2013c) improved the statistical approach for the consistency test.

\section{Preference aggregation}

The 2 most useful approaches traditionally applied to the aggregate individual preference are the aggregation of individual judgements (AIJ) and the aggregation of individual priorities (AIP) (Ramanathan \& Ganesh, 1994, and Forman \& Peniwati, 1998). For both the AIJ and AIP aggregation approaches, decision 
makers commonly use the weighted arithmetic mean (WAM) and the weighted geometric mean (WGM) to determine group preferences; it has been proven that these approaches satisfy the Pareto optimality principle (Aczel \& Saaty, 1983 and Forman \& Peniwati, 1998). The mathematical programming method is also used to group prioritization processes for different preference structures. Xu et al. (2011b) and Ma et al. (2006) proposed the optimization approach for different integrated preference structures. Fan (2006) developed a goal programming approach to derive the group preference, which has the smallest gap from each decision maker's opinion. Xu and Cai (2012) used some mathematical programming models to determine the reasonable weight of each decision maker, essentially extending the mathematical averaging aggregation methods. Hosseinian (2012) proposed a new linear programming method in order to generate weights in the analytic hierarchy process. Other preference aggregation methods were also proposed for the AHP-GDM. Srdjevic and Srdjevic (2012) designed the MGPS algorithm using multi-criteria evaluation scores for the synthesis of the local priority vectors in a local context. Bernasconi (2014) compared and evaluated the properties of 5 aggregation methods, and proposed approaches to correct the systematic distortions. Chao et al, (2017) proposed an optimization model to integrate multiplicative and fuzzy preferences.

\section{Consensus reaching}

The consensus-improving model is a fundamental concept and ultimate aim of the GDM, which is one of most significant issues in the GDM (Moreno-Jiménez et al., 2014). The group priority vector need to achieve agreement between the decision makers is implicated in the estimation of the problem. In most cases, there are conflicts among the decision makers; therefore, many existing methods have been proposed to improve AHPGDM consensus degree. Altuzarra et al. (2010) studied the consensus process by means of a posteriori information using the Bayesian perspective. Dong et al. (2010) proposed iteration-adjusting algorithms for consensus reaching based on the row geometric mean prioritization method. $\mathrm{Wu}$ and $\mathrm{Xu}$ (2012) provided several parameter adjustment and iteration models that could be used if an acceptable degree of individual consistency was given. Pedrycz and Song (2011) developed consensus models based on information granularity. Escobar et al. (2007) proposed a procedure that obtained the holistic importance of each alternative and employed it for consensus reaching.

Consistency and other performance evaluation criteria are important topics in the AHP-GDM, but there are no agreement indices for the consensus metric in the above issues. The popular measurements for all priority derivation methods for a single PCM are the generalized Euclidean distance (ED) and minimum violations (MV) approaches introduced by Golany and Kress (1993); these approaches are widely used by researchers (Srdjevic, 2005; Mikhailov \& Singh, 1999). Lin and Kou (2015) proposed the Bayesian revision method for improving the individual inconsistency of the PCM before using the AIJ and AIP. Dong et al. (2015) created a new individual consistency index and used the individual numerical scale in the AHP-GDM.

Existing aggregation and consensus models that have been used in the AHP-GDM work with the mathematical expectation of weights given in advance that presented the importance of different decision makers. However, the main limitation of this method is how to assign reasonable weights so that the group preference is nearest to the individual preference. Another problem in many practical situations using the AHP-GDM is that the individual decision makers are not able to be compared with optimal modification in PCM for consensus reaching, which should be provided to decision makers for the search consensus. Furthermore, many methods (Wu \& Xu, 2012, Xu \& Cai, 2012, Dong et al., 2012) for consensusreaching models in the AHP-GDM implement iterative algorithms in order to obtain the consensus results until a pre-defined consensus index is met. This makes it difficult to employ the model to practical fields due to computational complexity of interactive preference modification mechanism in these models.

Given this background, we present 2 new preference aggregation and consensus models that avoid the previously mentioned limitations concerning weights that are uncertainly assigned and the iteration complexity. For the aggregation of the individual judgments, we employed a quadratic programming model to optimize the total minimum deviations between 
each individual priority vector and the group's priority vector. The model directly acquired the optimal group collective priority vector with the calculated weights. Then, we constructed a logarithmic linear equation and computed the adjusted value for the individual PCMs to reach the consensus. The proposed priority vector returns to every decision maker and gives them adjusted PCM information to help them evaluate their judgements within the individual accepted interval scale.

The remainder of this paper is arranged as follows. The next section provides preliminary knowledge. Section III describes the optimization models for the preference aggregation in the AHP-GDM problem related to individual judgements and priorities. Next, section IV includes consensus-reaching models for the proposed optimization methods. In section $\mathrm{V}$, numerical examples are illustrated. Finally, concluding remarks are made in section VI.

\section{Preliminary knowledge}

The AHP is a multi-criteria decision-making approach that comes from both a theoretical and practical understanding. The essential issues relating to the AHP are the derivation of the priority vector and the studies on the consistency of a PCM. First, some definitions related to the AHP are introduced as follows:

Definition 1 (Saaty, 1980): A multiplicative preference relation on a set of alternatives $X=\left\{x_{1}, x_{2}, \ldots, x_{n}\right\}$ is represented by a matrix, $A=\left(a_{i j}\right)_{n \times n}$, where the entry of matrix $a_{i j}$ is identified on a 1-9 scale and interpreted as the ratio of the preference intensity of the alternatives' relative importance. If $a_{i j}=1$, the alternatives have the same preference and the $x_{i}$ dominat $x_{j}$ while $a_{i j}>1$. The multiplicative reciprocity is held in this matrix, i.e., $a_{i j} \times a_{j i}=1, \forall i, j \in 1,2, \ldots, n$.

The matrix which satisfies the multiplicative preference relation is also called a positive reciprocal matrix, and is identified as perfectly consistent if

$a_{i l}=a_{i j} \times a_{j l}$ for all $i, j, l \in\{1,2, \ldots, n\}$.

\section{Row geometric mean priority deviation method (RGMM)}

The priority vector $W=\left(w_{1}, w_{2}, \ldots, w_{n}\right)$ in the RGMM is formulated based on the nearest perfect consistent conditions where $a_{i j}$ is approximately equal to $\frac{w_{i}}{w_{j}}$, and the following optimization problem can obtain a unique solution (Crawford \& Williams, 1985):

$$
\begin{aligned}
& \text { Min } \quad \sum_{i=1}^{n} \sum_{j=i+1}^{n}\left(\ln a_{i j}-\ln w_{i}+\ln w_{j}\right)^{2} \\
& \text { S.t. } \quad w_{i} \geq 0, \sum_{i=1}^{n} w_{i}=1 .
\end{aligned}
$$

The solution of the above model can be computed as the following formula, which is essentially the geometric means of the rows of matrix $A$ :

$$
w_{i}=\frac{\sqrt[1 / n]{\prod_{j=1}^{n} a_{i j}}}{\sum_{i=1}^{n} \sqrt[1 / n]{\prod_{j=1}^{n} a_{i j}}}, i=1,2, \ldots, n
$$

$\mathrm{Xu}$ and Wei (1999) developed a method to improve the consistency by adjusting the iterations based on the RGMM as follows:

$$
a_{i j}^{(t+1)}=\left(a_{i j}^{(t)}\right)^{\theta}\left(\frac{w_{i}^{(t)}}{w_{j}^{(t)}}\right)^{1-\theta}
$$

where $t$ is the iteration time. The algorithm will converge after many iterations.

\section{AHP-GDM}

The GDM obtains the group preference by the aggregation of the individual judgements or preferences, which is difficult to accomplish with the discrepancy between the decisionmakers' opinions. The GDM encourages the integration of the individual views into a collective opinion. In the AHP-GDM, each decision maker will provide an individual PCM, and then employ aggregation rules for all individual PCMs. The most widely used methods are the AIJ and AIP.

1. The aggregation of the individual judgments

For the AIJ, the group judgement matrix aggregates the individual judgement matrices using the weighted geometric mean method. 
Assume $\left(\lambda_{1}, \lambda_{2}, \ldots, \lambda_{K}\right)$ is the weight vector of $K$ decision makers. In the group judgment matrix $A^{G}=\left(a i j_{i j}^{G}\right), i=1,2 \ldots, n ; j=1,2 \ldots, n$, where

$$
a_{i j}{ }^{G}=\prod_{k=1}^{K}\left(a_{i j}{ }^{k}\right)^{\lambda_{k}} .
$$

Then, the moderator uses one or a combined prioritization derivation method to obtain a group priority vector to rank alternatives.

\section{The aggregation of the individual priorities}

Let $W^{k}=\left(w_{1}^{k}, w_{2}^{k}, \ldots, w_{n}^{k}\right)^{T}$ be the individual priority vector derived from individual judgment matrix $A^{k}$ using a certain prioritization method and $\left(\lambda_{1}, \lambda_{2}, \ldots, \lambda_{K}\right)$ is the weight vector of $K$ decision makers. Then, the group priority vector obtained is $W^{G}=\left(w_{1}^{G}, w_{2}^{G}, \ldots, w_{n}^{G}\right)^{T}$, where

$$
w_{i}^{G}=\frac{\prod_{k=1}^{K}\left(w_{i}^{k}\right)^{\lambda_{k}}}{\sum_{i=1}^{n} \prod_{k=1}^{K}\left(w_{i}^{k}\right)^{\lambda_{k}}} .
$$

In addition, the WAMM is frequently used for the aggregation of the individual priorities vectors by $w_{i}^{G}=\sum_{k=1}^{K} \lambda_{k} w_{i}^{k}$. It easy to conclude that $\sum_{i=1}^{G} w_{i}^{G}=1$.

\section{Performance evaluation criteria}

In a single PCM, the geometric consistency index (Aguaron \& Jimenez, 2003) is a formalized inconsistency measure based on the RGMM. Jimenez (2015) extended the GCI to the group compatibility metric.

The geometric consistency index (GCI) is

$$
G C I=\frac{1}{(n-1)(n-2)} \sum_{i<j} \log ^{2}\left(\frac{a_{i j}^{(k)} w_{j}^{G}}{w_{i}^{G}}\right) .
$$

The group compatibility index (GGCI) is:

$$
G G C I=\sum_{k=1}^{K} \lambda_{k} \frac{1}{(n-1)(n-2)} \sum_{i<j} \log ^{2}\left(\frac{a_{i j}^{(k)} w_{j}^{G}}{w_{i}^{G}}\right) \text {. }
$$

Dong et al. (2010) proposed the geometric ordinal consensus index (GOCI) to measure the consensus degree. Their index is as follows:

$\operatorname{GOCI}\left(A^{(k)}\right)=\frac{1}{n} \sum_{i=1}^{n}\left|v^{(k)}-v^{G}\right|$,

where $v^{(k)}$ is the position of the ith alternative in priority.

$\mathrm{Wu}$ and $\mathrm{Xu}$ (2012) proposed a new consensus index, $G C I_{H}$, based on the Hadamard product of 2 matrices as follows:

$\operatorname{GCI}_{H}\left(A^{(k)}\right)=\frac{1}{n^{2}} \sum_{i=1}^{n} \sum_{j=1}^{n} a_{i j}^{(k)} a_{j i}^{G}$.

Aguarón and Moreno-Jiménez (2003) and Escobar et al. (2004) proposed the geometric consistency index for the AHP and AHP-GDM.

\section{A consensus model for group decision making}

Assume $K$ decision makers provide $K$ PCMs for group decision making, and their judgements represent multiplicative preference relations, as shown in Definition 1. Each decision maker compares each pair of $n$ alternatives using a 1-9 ratio scale. If all the individual PCMs

$\left(a_{i j}^{(k)}\right), i=1,2, \ldots n ; j=i+1, . ., n ; k=1,2, \ldots K \quad$ are consistent with the group judgement using the WGMM, then the group consensus is reached. In practical applications, there are conflicts among the decision makers' judgements. The group judgement should be close to the individual judgements or minimize the deviation in the individual PCMs and group judgment. In this section, we propose aggregation models based on the optimization insight. These models provide the optimization weights for the individual priority vectors, and the group priority vector has the least total matrix norm between the group priority vectors and individual priority vectors. Then, we employ logarithmic linear equations to calculate the least square solution in order to obtain an approximate consensus-reaching model in the AHP-GDM. The main differences between our method and existing models are the aggregation priority vectors from the optimization model and optimal weights.

Another innovation is the computation of the deviation factors by the logarithmic linear equations replacing the iterations algorithm. As 
there is no likelihood of confusion, in order to simplify the notation, the $k$ th decision maker will be denoted as $(k)$.

Suppose $\quad w^{(k)}=\left(w_{1}^{(k)}, w_{2}^{(k)}, \ldots, w_{n}^{(k)}\right) \quad$ is an individual priority vector of the $k$ th decision maker and $w_{i}^{G}=\sum_{l=1}^{K} x_{l} w_{i}^{l}$ is the group aggregation priority vector from the optimization weights based on the Frobenius norm of the matrix.

We minimize the deviation measured with the Frobenius norm from the group priority to individual preference vectors, which is the aggregation of the individual priority vectors by the optimization weights, and set the equivalence model formally as follows:

$\operatorname{Min} \sum_{k=1}^{K} \sum_{i=1}^{n}\left(w_{i}^{G}-w_{i}^{(k)}\right)^{2}$,

S.t. $\left\{\begin{array}{c}\sum_{k=1}^{K} x_{k}=1 \\ x_{k} \geq 0, k=1,2, \ldots, K\end{array}\right.$.

The Lagrange function of the above quadratic programming is as follows:

$L(x, \lambda)=\sum_{k=1}^{K} \sum_{i=1}^{n}\left(w_{i}^{G}-w_{i}^{(k)}\right)^{2}-\lambda\left(\sum_{k=1}^{K} x_{k}-1\right)$

where $\lambda$ is the Lagrange multiplier.

By taking the partial derivative with respect to $x_{k}(k=1,2, \ldots, K)$ and $\lambda$, and then setting these partial derivatives equal to 0 , the following sets of equations were obtained:

$\frac{\partial(L(x, \lambda))}{\partial \lambda}=\sum_{k=1}^{K} x_{k}-1=0$

and

$\frac{\partial(L(x, \lambda))}{\partial x_{j}}=2 \sum_{i=1}^{n}\left[\sum_{k=1}^{K}\left(w_{i}^{G}-w_{i}^{(k)}\right)\right] w_{i}^{(j)}-\lambda=0$

where $j=1,2, \ldots, K$.

The previous equations can be re-written as

$2 \sum_{i=1}^{n} \sum_{k=1}^{K}\left(w_{i}^{G} w_{i}^{(j)}-w_{i}^{(k)} w_{i}^{(j)}\right)-\lambda=0$

where $j=1,2, \ldots, K$

and

$2 \sum_{i=1}^{n} \sum_{k=1}^{K}\left(\sum_{l=1}^{K} x_{l} w_{i}^{(l)} w_{i}^{(j)}-w_{i}^{(k)} w_{i}^{(j)}\right)-\lambda=0$

http://www.sic.ici.ro where $j=1,2, \ldots, K$.

That is

$2 K \sum_{l=1}^{K} x_{l}\left(\sum_{i=1}^{n} w_{i}^{(l)} w_{i}^{(j)}\right)-2 \sum_{k=1}^{K} \sum_{i=1}^{n} w_{i}^{(k)} w_{i}^{(j)}-\lambda=0$

where $j=1,2, \ldots, K$.

We denote $J, H$, and $e$ as follows:

$$
\begin{aligned}
& J=\left(\sum_{k=1}^{K} \sum_{i=1}^{n} w_{i}^{(k)} w_{i}^{(1)}, \sum_{k=1}^{K} \sum_{i=1}^{n} w_{i}^{(k)} w_{i}^{(2)}, \ldots, \sum_{k=1}^{K} \sum_{i=1}^{n} w_{i}^{(k)} w_{i}^{(K)}\right)^{T} \\
& e=(1,1, \ldots, 1)^{T}, \text { and }
\end{aligned}
$$

$H=\left(\begin{array}{cccc}\sum_{i=1}^{n} K w_{i}^{(1)} w_{i}^{(1)} & \sum_{i=1}^{n} K w_{i}^{(2)} w_{i}^{(1)} & \ldots & \sum_{i=1}^{n} K w_{i}^{(K)} w_{i}^{(1)} \\ \sum_{i=1}^{n} K w_{i}^{(2)} w_{i}^{(1)} & \sum_{i=1}^{n} K w_{i}^{(2)} w_{i}^{(2)} & \ldots & \sum_{i=1}^{n} K w_{i}^{(K)} w_{i}^{(2)} \\ \ldots & \ldots & \ldots & \ldots \\ \sum_{i=1}^{n} K w_{i}^{(K)} w_{i}^{(1)} & \sum_{i=1}^{n} K w_{i}^{(K)} w_{i}^{(2)} & \ldots & \sum_{i=1}^{n} K w_{i}^{(K)} w_{i}^{(K)}\end{array}\right)_{K \mathrm{x} \mathrm{K}}$

Then, the previous equation sets can be rewritten in the above equation set in a matrix form as

$$
2 H x-2 J-\lambda e=0
$$

and

$2 \mathrm{Hee}^{T} \mathrm{X}-2 \mathrm{~J}-\lambda e=0$.

Next, we obtain

$\lambda=2 e^{T} H e-2 e^{T} J$

and

$x=H^{-1} J+H^{-1}\left(e^{T} H e-e^{T} J\right) e$.

Thus, the optimal priority vector of group decision making is

$$
\begin{aligned}
& w^{G}=x^{T}\left(w^{(1)}, w^{(2)}, \ldots, w^{(K)}\right)^{T} \\
& =\left(H^{-1} J+H^{-1}\left(e^{T} H e-e^{T} J\right) e\right)^{T}\left(w^{(1)}, w^{(2)}, \ldots, w^{(K)}\right)^{T}
\end{aligned}
$$

The next subsection describes the consensus model for the AHP-GDM based on the RGMM. Assume the individual PCM is $A^{k}$, and $e_{i j}^{(k)}$ is the adjustment derivation for $a_{i j}^{(k)}$.

$$
A^{k}=\left(\begin{array}{cccc}
a_{11}^{(k)} & a_{12}^{(k)} & \cdots & a_{1 n}^{(k)} \\
a_{21}^{(k)} & a_{22}^{(k)} & \cdots & a_{2 n}^{(k)} \\
\vdots & \vdots & \ddots & \vdots \\
a_{n 1}^{(k)} & a_{n 2}^{(k)} & \cdots & a_{n n}^{(k)}
\end{array}\right),
$$

the adjusted PCM is $A^{k,{ }^{*}}=\left(a_{i j}^{(k)}\right)^{\theta}\left(e_{i j}^{k}\right)^{1-\theta}$, 
$A^{k, *}=\left(\begin{array}{cccc}\left(a_{11}^{(k)}\right)^{\theta}\left(e_{1(}^{(k)}\right)^{1-\theta} & \left(a_{12}^{(k)}\right)^{\theta}\left(e_{12}^{(k)}\right)^{1-\theta} & \cdots & \left(a_{1 n}^{(k)}\right)^{\theta}\left(e_{1 n}^{(k)}\right)^{1-\theta} \\ \left(a_{21}^{(k)}\right)^{\theta}\left(e_{21}^{(k)}\right)^{1-\theta} & \left(a_{22}^{(k)}\right)^{\theta}\left(e_{22}^{(k)}\right)^{1-\theta} & \cdots & \left(a_{2 n}^{(k)}\right)^{\theta}\left(e_{2 n}^{(k)}\right)^{1-\theta} \\ \vdots & \vdots & \ddots & \vdots \\ \left(a_{n 1}^{(k)}\right)^{\theta}\left(e_{n 1}^{(k)}\right)^{1-\theta} & \left(a_{n 2}^{(k)}\right)^{\theta}\left(e_{n 2}^{(k)}\right)^{1-\theta} & \cdots & \left(a_{n n}^{(k)}\right)^{\theta}\left(e_{n n}^{(k)}\right)^{1-\theta}\end{array}\right)$

and its priority vector is

$w^{k^{k *}}=\left(\prod_{j=1}^{n}\left[\left(a_{1 j}^{(k)}\right)^{\theta}\left(e_{1 j}^{(k)}\right)^{1-\theta}\right], \prod_{j=1}^{n}\left[\left(a_{2 j}^{(k)}\right)^{\theta}\left(e_{2 j}^{(k)}\right)^{1-\theta}\right], \cdots, \prod_{j=1}^{n}\left[\left(a_{k j}^{(k)}\right)^{\theta}\left(e_{k j}^{(k)}\right)^{1-\theta}\right]\right)$

Let $w^{k, *}=w^{G}=x^{T}\left(w^{(1)}, w^{(2)}, \ldots, w^{(K)}\right)^{T}$, and take the logarithm for the proposed equation, that is

$$
\begin{aligned}
& \left(\sum_{j=1}^{n} n^{-1}\left(\theta \ln a_{1 j}^{(k)}+(1-\theta) \ln e_{1 j}^{(k)}\right), \sum_{j=1}^{n} n^{-1}\left(\theta \ln a_{2 j}^{(k)}+(1-\theta) \ln e_{2 j}^{(k)}\right),\right. \\
& \left.\ldots, \sum_{j=1}^{n} n^{-1}\left(\theta \ln a_{K j}^{(k)}+(1-\theta) \ln e_{K j}^{(k)}\right)\right)=\left(\ln w_{1}^{G}, \ln w_{2}^{G}, \ldots, \ln w_{K}^{G}\right)
\end{aligned}
$$

We can deal

with $e_{i j}^{(k)}=\frac{s_{i}^{(k)}}{s_{j}^{(k)}}, i, j=1,2, \ldots, n ; k=1,2, \ldots K$

using the separable representations theory from Bernasconi et al.(2010). The adjustments of the individual PCMs (Altuzarra 2007, 2010) indicate that the deviations follow Gaussian distributions, that is $e_{i j}^{(k)} \propto N\left(\frac{s_{i}^{(k)}}{s_{j}^{(k)}}, \sigma^{2}\right)$, $i, j=1,2, \ldots, n ; k=1,2, \ldots K$, and the estimator of variance $\sigma^{2}$ is $\frac{1}{n^{2}-3 n} \sum_{i, j=1}^{n} \hat{\varepsilon}_{i j}^{2}$,where $\hat{\varepsilon}_{i j}=\ln a_{i j}-\left(\ln w_{i}-\ln w_{j}\right)-\beta\left(\ln w_{i}-\ln w_{j}\right)^{3}$ (Bernasconi et al., 2010).

$$
\left(\sum_{j=1}^{n} \ln e_{1 j}^{(k)}, \sum_{j=1}^{n} \ln e_{2 j}^{(k)}, \ldots, \sum_{j=1}^{n} \ln e_{K j}^{(k)}\right)=(1-\theta)^{-1}\left(n \ln w_{1}^{G}-\theta \ln w_{1}^{(k)},\right.
$$$$
\left.n \ln w_{2}^{G}-\theta \ln w_{2}^{(k)}, \ldots, n \ln w_{K}^{G}-\theta \ln w_{K}^{(k)}\right)
$$

Consequently, we obtain

$$
\begin{gathered}
\ln e_{i j}^{(k)}=\sum_{j=1}^{n}\left(\ln s_{i}^{(k)}-\ln s_{j}^{(k)}\right) \ln \sigma_{i j}^{2} ; \\
\quad i=1,2, \ldots, K \text { and } \sigma_{i j}^{2} \text { are the deviation }
\end{gathered}
$$
factors.

$$
\begin{aligned}
& \left(\sum_{j=1}^{n}\left(\ln s_{1}^{(k)}-\ln s_{j}^{(k)}\right) \ln \sigma_{1 j}^{2}, \sum_{j=1}^{n}\left(\ln s_{2}^{(k)}-\ln s_{j}^{(k)}\right) \ln \sigma_{2 j}^{2}, \ldots,\right. \\
& \left.\sum_{j=1}^{n}\left(\ln s_{K}^{(k)}-\ln s_{j}^{(k)}\right) \ln \sigma_{K j}^{2}\right)=(1-\theta)^{-1}\left(n \ln w_{1}^{G}-\theta \ln w_{1}^{(k)},\right. \\
& \left.n \ln w_{2}^{G}-\theta \ln w_{2}^{(k)}, \ldots, n \ln w_{K}^{G}-\theta \ln w_{K}^{(k)}\right)
\end{aligned}
$$

As the above equality can be changed into $n$ unknown linear equations, it can be easily solved.

$$
\begin{aligned}
& \sum_{j=1}^{n}\left(\ln s_{1}^{(k)}-\ln s_{j}^{(k)}\right) \ln \sigma_{1 j}^{2}=(1-\theta)^{-1}\left(n \ln w_{1}^{G}-\theta \ln w_{1}^{(k)}\right) \\
& \sum_{j=1}^{n}\left(\ln s_{2}^{(k)}-\ln s_{j}^{(k)}\right) \ln \sigma_{2 j}^{2}=(1-\theta)^{-1}\left(n \ln w_{2}^{G}-\theta \ln w_{2}^{(k)}\right)
\end{aligned}
$$

$\sum_{j=1}^{n}\left(\ln s_{K}^{(k)}-\ln s_{j}^{(k)}\right) \ln \sigma_{2 j}^{2}=(1-\theta)^{-1}\left(n \ln w_{K}^{G}-\theta \ln w_{K}^{(k)}\right)$

We need to show that the coefficient matrix of the above equations set is a singular matrix without regard to the deviation factors, thus, $\ln \sigma_{i j}^{2}$ is the adjusted factors of the least square norm solution by the Moore-Penrose inverse matrix. Therefore, we can obtain the solution $\left(s_{i}^{k}\right)_{K \times 1}$ as follows:

$$
\begin{aligned}
& \left(\begin{array}{c}
s_{1}^{k} \\
s_{2}^{k} \\
\vdots \\
s_{K}^{k}
\end{array}\right)=\exp \left[\left(\begin{array}{cccc}
K-1 & -1 & \cdots & -1 \\
-1 & K-1 & \cdots & -1 \\
\vdots & \vdots & \ddots & \vdots \\
-1 & -1 & \cdots & K-1
\end{array}\right)^{+}(1-\theta)^{-1}\left(\begin{array}{c}
n \ln w_{1}^{G}-\theta \ln w_{1}^{(k)} \\
n \ln w_{2}^{G}-\theta \ln w_{2}^{(k)} \\
\vdots \\
n \ln w_{K}^{G}-\theta \ln w_{K}^{(k)}
\end{array}\right)\right] \\
& =\exp \left[( \begin{array} { c c c c } 
{ K - 1 } & { - 1 } & { \cdots } & { - 1 } \\
{ - 1 } & { K - 1 } & { \cdots } & { - 1 } \\
{ \vdots } & { \vdots } & { \ddots } & { \vdots } \\
{ - 1 } & { - 1 } & { \cdots } & { K - 1 }
\end{array} ) ^ { + } ( 1 - \theta ) ^ { - 1 } \left(n \ln \left(H^{-1} J+H^{-1}\left(e^{T} H e-e^{T} J\right) e\right)^{T}\right.\right.
\end{aligned}
$$$$
\left.\left.\left(w^{(1)}, w^{(2)}, \ldots, w^{(K)}\right)^{T}-\theta \ln \left(w_{1}^{(k)}, w_{2}^{(k)}, \ldots, w_{K}^{(k)}\right)^{T}\right)\right]
$$

where $k=1,2, \ldots, K$, and $A^{+}$is the MoorePenrose inverse matrix.

If there are 3 alternatives, the consensus model can accurately calculate the deviation factors and obtain a perfect consensus preference rank since the numbers of unknown variables are equal to the equations. In other cases, we can adjust the PCMs by the proposed method to obtain an approximate group consensusreaching model.

\section{Remark 1:}

With respected to the adjusted PCMs, the subjective judgments of the decision makers must be considered as the major criteria to solve the group decision-making questions. The proposed methods can give decision makers the group information to adjust the comparative degree using Saaty's 1-9 ratio scale. If the adjusted interval is less than a small accepted range, the adjustment can be implemented. Our model is an effective method when the decision makers agree to adjust the individual PCMs. 


\section{Remark 2:}

The main improvement of our method is its ability to directly calculate the approximate consensus priority vector based on the linear equation sets rather than reaching consensus by iteration. The computing complexity can be greatly decreased, and the priority vector is an optimization solution that is close to the individual priority vectors. Since errors, $e_{i j}$, are recognized as an important source of possible inconsistencies in the AHP (Bernasconi, 2010), we supposed that the errors obeyed the Gaussian-distribution-applied MoorePenrose-generated inverse. The polynomial approximation and other methods can also be used to solve the deviation, and the Tikhonov regularization method can replace the Moore-Penrose-generated inverse for a more accurate solution.

\section{Examples}

In this section, we show the advantages of the proposed method with 2 illustrative examples.

First, we use 4 steps to implement our consensus model. The steps are as follows:

Step1: Calculate the optimal weights, $x$, and group priority vector, $w^{G}$.
Step2: Compute the adjusted derivations for each decision maker using the separable representations theory,

$$
\left(s_{1}^{(k)}, s_{2}^{(k)}, \ldots, a_{n}^{(k)}\right)^{T} \text {. }
$$

Step3: Calculate adjustment matrix $A^{K, *}$.

Step4: Calculate the increment $A^{K}-A^{K, *}$, then return the adjustment matrix as decisionmaking information to each decision maker. If the decision makers accept the adjustment interval, the adjustment can be implemented, otherwise, go to step 1 .

Step5: Do not stop the adjustment until all the decision makers accept the group priority vector.

Example 1. Consider the AHP-group decisionmaking questions below, which were investigated by Ramanathan et al. (1994).

Table 1 represents the PCMs of 2 decision makers and the priority vectors by the RGMM. The GCI index of the PCM of the $1^{\text {st }}$ decision maker means that the consistency is unacceptable and needs to be adjusted since the consistency is larger than 0.37 , which is the threshold corresponding toCR=0.1. The priority vectors of the 2 decision makers have a controversy, which ranks as $5 \succ 3 \succ 4 \succ 1 \succ 2$ and $4 \succ 2 \succ 5 \succ 3 \succ 1$, respectively.

Table 1. PCMs of 2 decision makers and priority vector

\begin{tabular}{|l|l|l|l|l|l|}
\hline C1 & C2 & C3 & C4 & C5 & Priority \\
\hline 1,1 & $1 / 3,1 / 3$ & $1 / 3,1$ & $1 / 9,1 / 3$ & $1 / 5,1 / 7$ & $0.0454(5), 0.0601(4)$ \\
\hline & 1,1 & 1,3 & $1 / 3,1$ & $1 / 3,1 / 5$ & $0.1212(3), 0.1547(2)$ \\
\hline & & 1,1 & $1 / 3,1 / 3$ & $1 / 3,1 / 7$ & $0.1212(4), 0.0601(5)$ \\
\hline & & & 1,1 & $3,1 / 5$ & $0.4528(1), 0.1547(3)$ \\
\hline & & & & 1,1 & $0.2594(2), 0.5704(1)$ \\
\hline & & & & & GCI $=0.386$ GCI $=0.234$ \\
\hline
\end{tabular}


Table 2 shows the adjusted results $A^{K, *}$ by our proposed method. The 2 priority vectors have the same rank, and the GCI index is simultaneously less than 0.37 .

Table 2. Adjust matrix by our methods

\begin{tabular}{|l|l|l|l|l|l|}
\hline C1 & C2 & C3 & C4 & C5 & Priority \\
\hline $1.0000,1.0000$ & $0.4663,0.6246$ & $0.5324,0.7396$ & $0.2555,0.4019$ & $0.2934,0.4891$ & $0.0790(5), 0.1175(5)$ \\
\hline & $1.0000,1.0000$ & $1.1418,1.1842$ & $0.5479,0.6435$ & $0.4170,0.5686$ & $0.1561(3), 0.1764(3)$ \\
\hline & & $1.0000,1.0000$ & $0.4799,0.5434$ & $0.3652,0.6613$ & $0.1368(4), 0.1588(4)$ \\
\hline & & & $1.0000,1.0000$ & $1.6421,0.8835$ & $0.3324(1), 0.2742(1)$ \\
\hline & & & & $1.0000,1.0000$ & $0.2957(2), 0.2730(2)$ \\
\hline & & & & & GCI=0.352 GCI=0.363 \\
\hline
\end{tabular}

Now, we can calculate the deviation matrix $\left(e_{i j}^{(k)}\right)$ as follows. It illuminates the adjustment process, which can be changed in every entry by the proposed model. For example, the decision maker should consider alternative 1 versus alternative 4 , and compare them again; if the adjustment of -0.1444 can be accepted, they can change their original judgement. The decision makers need to check on their adjustment and then update the corresponding ratio scale values by means of the following deviation matrices.

$D_{1}=\left(\begin{array}{ccccc}0.0000 & -0.1329 & -0.1990 & -0.1444 & -0.0934 \\ 0.8552 & 0.0000 & -0.1418 & -0.2146 & -0.0837 \\ 1.1215 & 0.1242 & 0.0000 & -0.1446 & -0.0319 \\ 5.0857 & 1.1749 & 0.9162 & 0.0000 & 1.3579 \\ 1.5917 & 0.6020 & 0.2620 & -0.2759 & 0.0000\end{array}\right)$

$D_{2}=\left(\begin{array}{ccccc}0.0000 & -0.2913 & 0.2604 & -0.0686 & -0.3462 \\ 0.8552 & 0.0000 & 1.8158 & 0.3565 & -0.3686 \\ -0.3520 & -0.5111 & 0.0000 & -0.2101 & -0.5181 \\ 0.5120 & -0.5540 & 1.1598 & 0.0000 & -0.6835 \\ 4.9554 & 3.2412 & 5.4877 & 3.8682 & 0.0000\end{array}\right)$

The example shows that the a posteriori information can be provided to decision makers, which can help effectively negociation with each other and reach a consensus through the targeted adjustment of the comparative ratio scales.
Example 2.The PCMs of 3 decision makers are represented below; these were investigated by Yeh et al. (2001). The 3 individual PCMs are shown below:

$A_{1}=\left(\begin{array}{ccccc}1 & 5 & 7 & 3 & 1 / 3 \\ 1 / 5 & 1 & 3 & 1 / 3 & 1 / 5 \\ 1 / 7 & 1 / 3 & 1 & 1 / 7 & 1 / 9 \\ 1 / 3 & 3 & 7 & 1 & 1 / 3 \\ 3 & 5 & 9 & 3 & 1\end{array}\right)$

$A_{2}=\left(\begin{array}{ccccc}1 & 1 / 3 & 7 & 1 / 2 & 3 \\ 3 & 1 & 3 & 1 & 5 \\ 1 & 1 / 3 & 1 & 1 / 3 & 3 \\ 2 & 1 & 3 & 1 & 5 \\ 1 / 3 & 1 / 5 & 1 / 3 & 1 / 5 & 1\end{array}\right)$

and $A_{3}=\left(\begin{array}{ccccc}1 & 7 & 5 & 4 & 3 \\ 1 / 7 & 1 & 1 / 3 & 1 / 4 & 1 / 5 \\ 1 / 5 & 3 & 1 & 1 / 3 & 1 / 4 \\ 1 / 4 & 4 & 3 & 1 & 1 \\ 1 / 3 & 5 & 4 & 1 & 1\end{array}\right)$

It can be observed that $A_{2}$ has a significant difference relative to $A_{1}$ and $A_{3}$. For example, alternative 1 versus alternative 2, where $A_{1}$ and $A_{3}$ are 5 and 7 respectively, but $A_{2}$ is $1 / 3$. 
Table 3. Comparison results with other methods

\begin{tabular}{|c|c|c|c|c|c|c|c|c|c|c|c|c|}
\hline & \multicolumn{4}{|c}{ RGMM } & \multicolumn{4}{c|}{ WU \& XU(2012) } & \multicolumn{4}{c|}{ Our method } \\
\hline & $A_{1}$ & $A_{2}$ & $A_{3}$ & $A^{G}$ & $A_{1}$ & $A_{2}$ & $A_{3}$ & $A^{G}$ & $A_{1}$ & $A_{2}$ & $A_{3}$ & $A^{G}$ \\
\hline$W 1$ & 0.2813 & 0.1418 & 0.4878 & 0.3532 & 0.3160 & 0.3149 & 0.4669 & 0.3722 & 0.2423 & 0.2059 & 0.2500 & 0.2469 \\
\hline$W 2$ & 0.0695 & 0.3497 & 0.0436 & 0.1191 & 0.0755 & 0.1360 & 0.0492 & 0.0822 & 0.1683 & 0.2116 & 0.1634 & 0.1662 \\
\hline$W 3$ & 0.0321 & 0.1312 & 0.0809 & 0.0808 & 0.0432 & 0.0902 & 0.0788 & 0.0691 & 0.1434 & 0.1905 & 0.1581 & 0.1573 \\
\hline$W 4$ & 0.1590 & 0.3217 & 0.1780 & 0.2447 & 0.1823 & 0.2684 & 0.1863 & 0.2177 & 0.2049 & 0.2122 & 0.2062 & 0.2063 \\
\hline W5 & 0.4581 & 0.0555 & 0.2098 & 0.2022 & 0.3837 & 0.1905 & 0.2187 & 0.2587 & 0.2411 & 0.1798 & 0.2223 & 0.2234 \\
\hline Rank & 24531 & 31425 & 15432 & 14532 & 14532 & 14523 & 15432 & 14532 & 14532 & 32415 & 14532 & 14532 \\
\hline$G C I_{H}$ & 1.4303 & 2.1728 & 1.3192 & 1.0000 & 1.0526 & 1.0905 & 1.0889 & 1.0000 & 1.0067 & 1.0304 & 1.0105 & 1.0000 \\
\hline
\end{tabular}

Our modified method and comparative results can be verified in Table 3, and the rank is the same as that found with Wu and Xu's approach (2012). The $G_{H} I_{H}$ index for group decision making is also presented. The results show that both Wu and Xu's approach and our method have a consistency corresponding to a predefined threshold. Our model has a higher consensus than that of $\mathrm{Wu}$ and $\mathrm{Xu}$. $\mathrm{Wu}$ and Xu's approach needed to be modified 4,14,2 times while our results were not iterated and directly obtained.

\section{Conclusion}

In this paper we propose an optimization model for the obtaining group preference employed in AHP-group decision making that is relative to the AIP with pre-defined fixed weights (as mainly adopted in previous studies). The consensus-reaching method using the proposed optimizations model was indicated in order to present decision makers with how to adjust the individual PCMs for consensus reaching without interactive modification of individual PCM. It can give a criterion that can help to adjust judgments for individual actors, and the complexity is largely reduced since the equation sets are employed.

Two illustrative examples were implemented to show the effectiveness of our method. The results show that our method can also obtain consensus reaching in the AHP-GDM.
Furthermore, the proposed platform is easy to design as an intelligent system applied to provide decision support for AHP-GDM due to consensus reaching process is an intelligent computing process by means of equation sets solved by given analytic solution. the intelligent consensus reaching will help managers to obtain consensus group preference and select optimal alternative. Therefore, with rapid development of internet and mobile computing tools and platforms (Filip, 2014; Candea and Filip, 2016; Perez et al, 2010; Chao and Peng, 2017), the proposed method can be employed in more applications which need to handle large scale group decision making problems since decreasing computing process by means of the intelligent consensus reaching process.

\section{Acknowledgements}

This research has been partially supported by grants from the National Natural Science Foundation of China (\#71325001, \#71471149 and \#71433001), and Major project of the National Social Science Foundation of China (\# 15ZDB153).

\section{REFERENCES}

1. Aguarón, J. \& Moreno-Jiménez J. M.(2003) The geometric consistency index Approximated thresholds. European Journal of Operational Research 147(1) 137-145. 
2. Altuzarra, A., Moreno-Jiménez J. M. \& M. Salvador (2007) A Bayesian priorization procedure for AHP-group decision making. European Journal of Operational Research 182(1) 367-382.

3. Altuzarra, A., Moreno-Jiménez J. M. \& Salvador M., (2010). Consensus Building in AHP-Group Decision Making:A Bayesian Approach, Operations Research, 58(6) 1755-1773.

4. Bernasconi, M., Choirat C. \& Seri R., (2010). The Analytic Hierarchy Process and the Theory of Measurement. Management Science, 56(4) 699-711.

5. Bernasconi M., Choirat C. \& Seri R., (2014) Empirical properties of group preference aggregation methods employed in AHP: Theory and evidence, European Journal of Operational Research 232: 584-592.

6. Candea, C. \& Filip, F.G. (2016). Towards intelligent collaborative decision support platforms. Studies in Informatics and Control, 25(2), 143-152.

7. Chiclana F., Mata F., Martínez L. HerreraViedma E. \& Alonso S.,(2008) Integration of a consistency control module within a consensus decision making model, International Journal of Uncertainty, Fuzziness and Knowledge-Based Systems 16: 35-53.

8. Chao X.R., Kou G., \& Peng Y. (2017) A Similarity Measure-based Optimization Model for Group Decision Making with Multiplicative and Fuzzy Preference Relations. International Journal of Computers Communications \& Control. 12(1) 26-40.

9. Chao X.R. \& Peng Y. (2017) A Costsensitive Multi-Criteria Quadratic Programming Model for Imbalanced Data. Journal of the Operational Research Society. DOI: 10.1057/s41274-017-0233-4.

10. Cho Y.G. \& Cho K.T., (2008). A loss function approach to group preference aggregation in the AHP, Computers \& Operations Research 35 (2008) $884-892$.

11. Condon E., Golden B. \& Wasil B. (2003) Visualizing group decisions in the analytic hierarchy process. Computers \& Operations Research 30 (2003) 1435-1445.

12. Crawford G. \& Williams C. (1985). A note on the analysis of subjective judgment matrices. Journal of Mathematical Psychology, 29, 387-405.

13. Crawford, G. B. (1987). The geometric mean procedure for estimating the scale of a judgement matrix. Mathematical Modelling, 9, 327-334.

14. Dong Y. C., Fan Z. P. \& Yu S., (2015) Consensus Building in a Local Context for the AHP-GDM With the Individual Numerical Scale and Prioritization Method, IEEE Transactions on Fuzzy Systems, 23(2)354-368.

15. Dong Y. C., Zhang G. Q., Hong W. C. \& $\mathrm{Xu}$ Y. F., (2010)Consensus models for AHP group decision making under row geometric mean prioritization method, Decision Support Systems, vol. 49, 281-289, 2010.

16. Ergu D., Kou G., Peng Y. \& Shi Y. (2011). A simple method to improve the consistency ratio of the pair-wise comparison matrix in ANP. European Journal of Operational Research, 213, 246-259.

17. Escobar M.T., Aguaron J. \& MorenoJimenez J.M., (2004) A note on AHP group consistency for the row geometric, European Journal of Operational Research 153, 318-322.

18. Escobar M.T. \& Moreno-Jiménez J.M. (2007) Aggregation of Individual Preference Structures in Ahp-Group Decision Making, Group Decision and Negotiation 16: 287-301.

19. Escobar M. T. \& Aguarón J., MorenoJiménez J.M., (2015) Some extensions of the precise consistency consensus matrix, Decision Support Systems 74, 67-77.

20. Fan Z.P., Ma J., Yan-Ping Jiang, YongHong Sun, \& Louis Ma, (2006) A goal programming approach to group decision making based on multiplicative preference relations and fuzzy preference relations, European Journal of Operational Research 174, 311-321. 
21. Filip F.G. (2014) A Decision-Making Perspective for Designing and Building Information Systems, International Journal of Computers Communications \& Control. 7 (2) : 264-272.

22. Forman F. \& Peniwati K. (1998) Aggregating individual judgments and priorities with the Analytic Hierarchy Process, European Journal of Operational Research 108 (1998) 165-169.

23. Golany \& Kress (1993) A multicriteria evaluation of methods for obtaining weights from ratio-scale matrices. European Journal of Operational Research, 69, 210-220.

24. Hosseinian, S.S., Navidi H. \& Hajfathaliha A., (2012) A New Linear Programming Method for Weights Generation and Group Decision Making in the Analytic Hierarchy Process, Group Decision Negotiation (2012) 21:233-254.

25. Kou G., Ergu D., Lin C. \& Chen Y., (2016). Pairwise Comparison Matrix in Multiple Criteria Decision Making, Technological and Economic Development of Economy, DOI: http://dx.doi.org/10.3846/20294913.2016. 1210694 , 22(5): 738-765.

26. Kou G., Ergu D., Shi Y. \& Shi Y., (2014). An integrated expert system for fast disaster assessment. Computers \& Operations Research 42 (2014) 95-107.

27. Kou G. \& Lin C.S., (2014). A cosine maximization method for the priority vector derivation in AHP. European Journal of Operational Research (2014) 235(1), 25-232.

28. Lin C. S., Kou G. \& Ergu D. (2013a). An improved statistical approach for consistency test in AHP. Annals of Operations Research (2013) 211(1), 289-299.

29. Lin C. S., Kou, G. \& Ergu, D. (2013b). A statistical approach to measure the consistency level of the pairwise comparison matrix. Journal of the Operational Research Society (2014) 65(9) 1380-1386.
30. Lin, C. S., Kou, G., \& Ergu, D. (2013c). A heuristic approach for deriving the priority vector in AHP. Applied Mathematical Modelling, 37, 5828-5836.

31. Lin, C.S. \& Kou, G. (2015). Bayesian revision of the individual pair-wise comparison matrices under consensus inAHP-GDM, Applied Soft Computing http://dx.doi.org/10.1016/j.asoc.2015.02.041

32. Ma J., Fan Z. P., Jiang Y. P. \& Mao J. Y. (2006). An optimization approach to multiperson decision making based on different formats of preference information, IEEE Transactions on Systems Man and Cybernetics - Part A Systems and Humans, vol. 36, no. 5, pp. 876-889, Sep. 2006

33. Mikhailov \& Singh, (1999) Comparison analysis of methods for deriving priorities in the analytic hierarchy process. Proceedings of the IEEE International Conference on Systems, Man and Cybernetics, 1037-1042.

34. Mikhailov, L., (2004). Group prioritization in the AHP by fuzzy preference programming method, Computers \& Operations Research 31, 293-301.

35. Moreno-Jiménez J. M., Cardeñosa J., Gallardo C. \& Villa-Moreno M.A. de la, (2014). A new e-learning tool for cognitive democracies in the Knowledge Society, Computers in Human Behavior, 30, 9-18.

36. Pedrycz W. \& Song M., (2011). Analytic hierarchy process (AHP) in group decision making and its optimization with an allocation of information granularity, IEEE Transactions on Fuzzy Systems, vol. 19, no. 3, pp. 527-539, Jun. 2011.

37. Peláez, P. \& Lamata, M. (2003). A new measure of consistency for positive reciprocal matrices. Computers and Mathematics with Applications, 46, 1839-1845.

38. Perez, I. J., Cabrerizo, F. J., \& HerreraViedma, E. (2010). A mobile decision support system for dynamic group decision-making problems. IEEE Transactions on Systems, Man, and Cybernetics - Part A: Systems and Humans, 40(6), 1244-1256. 
39. Ramanathan R., \& Ganesh L.S. (1994)Group preference aggregation methods employed in AHP: An evaluation and an intrinsic process for deriving members' weightages, European Journal of Operational Research 79 (1994) 249-269.

40. Saaty, T. L. (1977). A scaling method for priorities in a hierarchical structure. Journal of Mathematical Psychology15 (3) 234-281.

41. Saaty T. L. (1977)A scaling method for priorities in hierarchical structures, Journal of Mathematical Psychology, vol. 15, 234-281, 1977.

42. Saaty T. L. (1980). The Analytic Hierarchy Process. New York, NY, USA: McGraw-Hill, 1980.

43. Saaty, T. L. (1986) Axiomatic foundation of the analytic hierarchy process. Management Science. 32(7) 841-855.

44. Saaty T.L. (1989) Group decision-making and the AHP, in: B.L. Golden, E.A. Wasil P.T. Harker (Eds.) The Analytic Hierarchy Process: Applications and Studies Springer-Verlag, New York, NY, 1989, pp. 59-67.

45. Srdjevic, B. (2005) Combining different prioritization methods in the analytic hierarchy process synthesis, Computers \& Operations Research 32 (2005) 1897-1919.

46. Srdjevic, B. \& Srdjevic Z. (2013). Synthesis of individual best local priority vectors in AHP-group decision making, Applied Soft Computing, vol. 13, 20452056, 2013.

47. Vaidya, O.S. \& Kumar, S., (2006). Analytic hierarchy process: An overview of applications, European Journal of Operational Research 169 (2006) 1-29.
48. Wang, Y.M., Parkan C. \& Luo Y., (2008). A linear programming method for generating the most favorable weights from a pairwise comparison matrix. Computers \& Operations Research 35 (2008) 3918-3930.

49. Wu Z.B. \& Xu J.P., (2012) A consistency and consensus based decision support model for group decision making with multiplicative preference relations, Decision Support Systems 52 (2012) 757-767.

50. Xu Z.S. \& Wei C.P., (1999) A consistency improving method in the analytic hierarchy process, European Journal of Operational Research 116 (1999) 443-449.

51. Xu Z.S. (2000). On consistency of the weighted geometric mean complex judgment matrix in AHP. European Journal of Operational Research 126(2000) 683-687.

52. Xu Z.S. \& Cai X.Q, (2011a). Group consensus algorithms based on preference relations, Information Sciences 181 (2011) 150-162.

53. Xu Z. S., Cai X. Q. \& Liu S. S. (2011b). Nonlinear programming model integrating different preference structures, IEEE Transactions on Systems Man and Cybernetics - Part A Systems and Humans, vol. 41, no. 1, 169-177, Jan. 2011.

54. Yeh J.M., Kreng B. \& Lin, C., (2001). A consensus approach for synthesizing the elements of comparison matrix in the Analytic Hierarchy Process, International Journal of Systems Science 32 (2001) 1353-1363. 